\title{
The No Harm Diet: A New Take on Ethical Diets
}

\section{Introduction}

Dietary habits of a population impacts the environment. To lessen the negative environmental effects, the No Harm Diet was designed and based on limiting harm to the organisms we receive our food from. Acceptable foods are plant or animal based and consumption does not harm the source.

Acceptable foods: Fruits, dairy products and unfertilized eggs Unacceptable foods: Meat, seafood, grains, legumes, and vegetables. These are considered unacceptable because these foods harm the organism or subsequent generation.
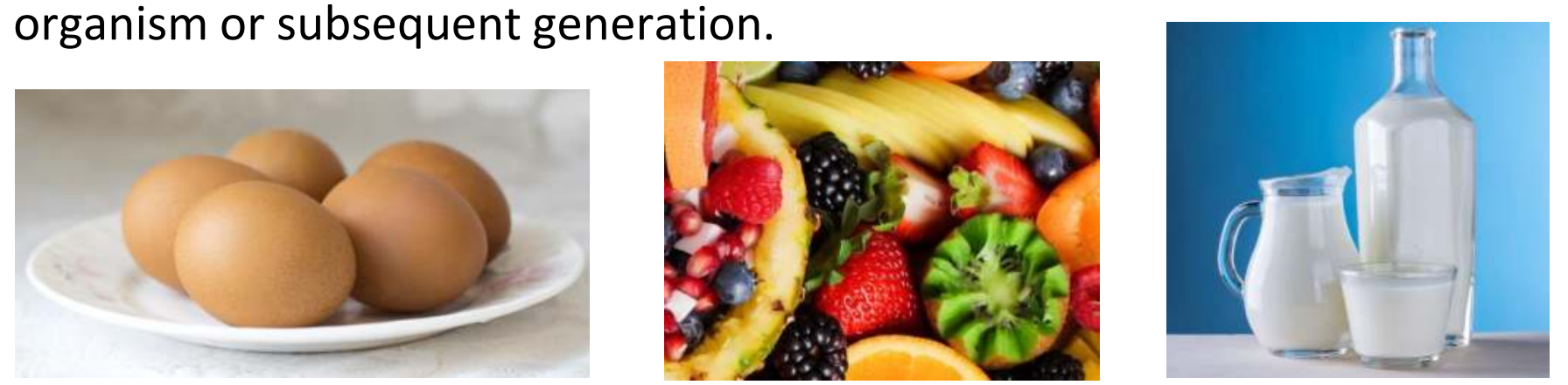

Objectives

Our aim was to determine if the No Harm Diet meets the Dietary

Reference Intake [DRI] recommendations

Created a one month meal plan

\section{Methods}

o $2000 \mathrm{kcal}$ per day

o 3 main meals and 2 snacks

o Resemble typical meals eaten by Canadians

- Generated nutrient intakes

o Nutritionist Pro software version 5.2.0

o Canadian Nutrient File

o Food selected to match type, cook and portion

- Descriptive statistics were generated

o SPSS

- Diet averages were compared

o DRI

o Average Canadian intakes

\begin{tabular}{|c|c|c|c|}
\hline \multicolumn{4}{|c|}{ Results } \\
\hline Mineral & $\begin{array}{l}\text { No Harm } \\
\text { Average }\end{array}$ & DRI [Men] & DRI [Women] \\
\hline $\begin{array}{l}\text { Sodium } \\
\mathrm{mg} / \text { day }\end{array}$ & 2126.34 & $1500[\mathrm{Al}]$ & $1500[\mathrm{Al}]$ \\
\hline $\begin{array}{l}\text { Calcium } \\
\mathrm{mg} / \text { day }\end{array}$ & 2021.90 & $800[E A R]$ & $800[E A R]$ \\
\hline $\begin{array}{l}\text { Potassium } \\
\mathrm{mg} / \text { day }\end{array}$ & 6062.82 & $4700[\mathrm{Al}]$ & $4700[\mathrm{Al}]$ \\
\hline $\begin{array}{l}\text { lodine } \\
\mu \mathrm{g} / \text { day }\end{array}$ & 0 & 95 [EAR] & 95 [EAR] \\
\hline $\begin{array}{l}\text { Iron } \\
\text { mg/day }\end{array}$ & 11.35 & $6[E A R]$ & 8.1 [EAR] \\
\hline $\begin{array}{l}\text { Iron [vegetarian] } \\
\text { mg/day }\end{array}$ & 11.35 & 10.8 [EAR] & $14.58[E A R]$ \\
\hline Phosphorus mg/da & 1939.50 & $580[$ EAR] & $580[E A R]$ \\
\hline $\begin{array}{l}\text { Zinc } \\
\mathrm{mg} / \text { day }\end{array}$ & 10.84 & 9.4 [EAR] & $6.8[E A R]$ \\
\hline Vitamin & $\begin{array}{l}\text { No Harm } \\
\text { Average }\end{array}$ & DRI [Men] & DRI [Women] \\
\hline $\begin{array}{l}\text { Vitamin A } \\
\text { IU/day }\end{array}$ & 9268.28 & 2083 [EAR] & 1667 [EAR] \\
\hline $\begin{array}{l}\text { Vitamin C } \\
\mathrm{mg} / \text { day }\end{array}$ & 613.53 & 75 [EAR] & $60[E A R]$ \\
\hline $\begin{array}{l}\text { Vitamin D } \\
\text { IU/day }\end{array}$ & 250.83 & $400[E A R]$ & $400[E A R]$ \\
\hline $\begin{array}{l}\text { Vitamin } \mathrm{E} \\
\mathrm{mg} / \text { day }\end{array}$ & 5.81 & 12 [EAR] & 12 [EAR] \\
\hline $\begin{array}{l}\text { Biotin } \\
\mu \mathrm{g} / \text { day }\end{array}$ & 0.12 & $30[\mathrm{Al}]$ & $30[\mathrm{Al}]$ \\
\hline $\begin{array}{l}\text { Folate } \\
\mu \mathrm{g} / \text { day }\end{array}$ & 439.93 & 320 [EAR] & $320[E A R]$ \\
\hline $\begin{array}{l}\text { Vitamin B12 } \\
\mu \mathrm{g} / \text { day }\end{array}$ & 5.98 & $2[E A R]$ & $2[E A R]$ \\
\hline
\end{tabular}

Percent Energy of the No Harm Diet Compared to the AMDR

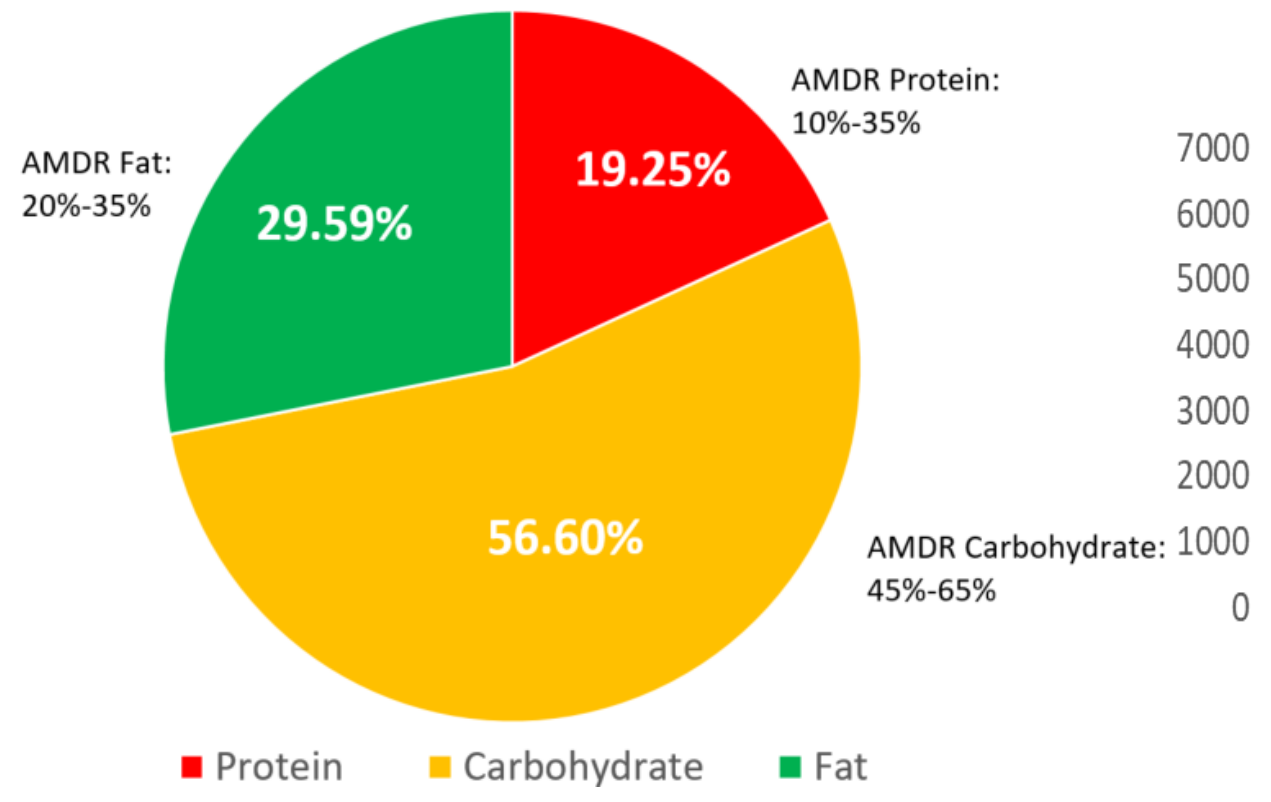

Mineral No Harm Averages Compared to Average Canadian Intake

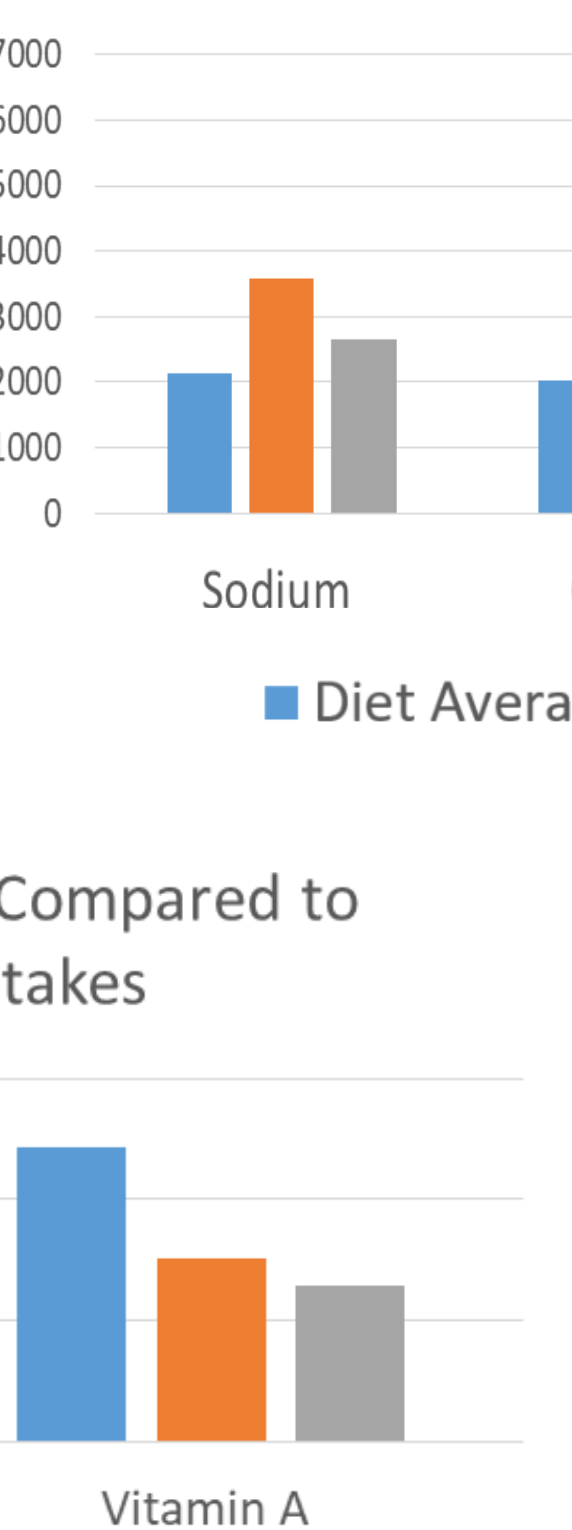

Mineral No Harm Averages Compared to Average Canadian Intake
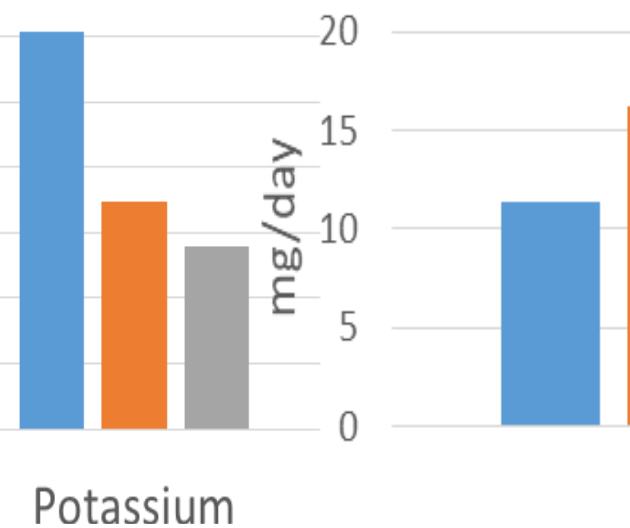

Iron

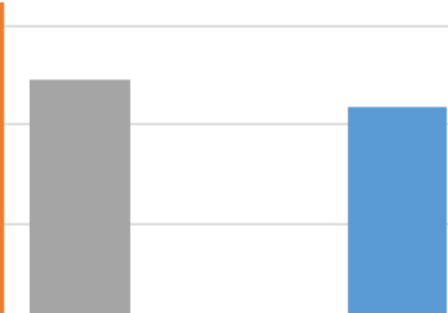

Calcium

Potassium

Zinc

Canadian Women Average
Vitamin No Harm Averages Compared to Average Canadian Intakes 1500

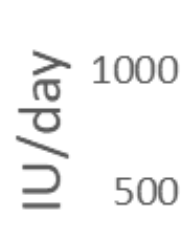$$
0
$$

Vitamin D
Vitamin No Harm Averages Compared to Average Canadian Intakes

60

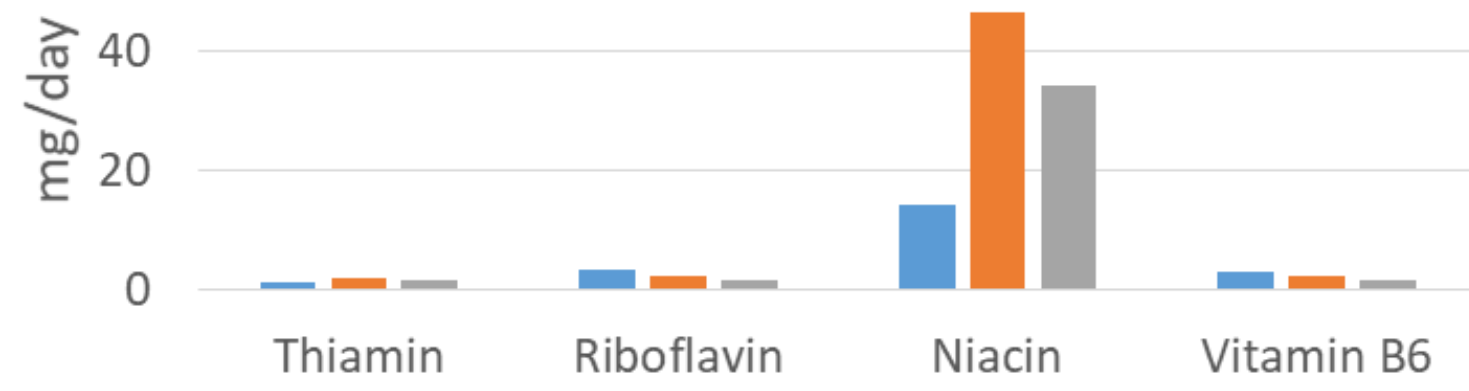

Diet Average $\quad$ Canadian Men Average $\quad$ Canadian Women Average

\section{Conclusion}

- Our findings suggested that the No Harm Diet is nutritionally adequate

- The No Harm Diet is a sustainable and environmentally friendly diet that could be a better equipped diet to help combat environmental change

Canada, H., Canadian Community Health Survey Cycle 2.2, Nutrition (2004). 2004. 1,2,3.

Canada, H., Dietary Reference Intakes Tables 2006.

www.canva.com
Natalie Wu: Natalie.wu@mail.mcgill.ca

Dr. Mark Lefsrud: mark.lefsrud@mcgill.ca Dr. Anne-Sophie Brazeau: anne-sophie.brazeau@mcgill.ca 